\title{
PERSPECTIVES
}

SCIENCE AND SOCIETY

\section{Advances in tuberculosis vaccine strategies}

Yasir A. W. Skeiky and Jerald C. Sadoff

Abstract|Tuberculosis (TB), an ancient human scourge, is a growing health problem in the developing world. Approximately two million deaths each year are caused by TB, which is the leading cause of death in HIV-infected individuals. Clearly, an improved TB vaccine is desperately needed. Heterologous prime-boost regimens probably represent the best hope for an improved vaccine regimen to prevent TB. This first generation of new vaccines might also complement drug treatment regimens and be effective against reactivation of TB from the latent state, which would significantly enhance their usefulness.

Mycobacterium tuberculosis, the causative agent of tuberculosis (TB), is the second leading infectious cause of mortality worldwide - M. tuberculosis has infected approximately two billion individuals in total, which is one third of the world's population ${ }^{1}$. Currently, TB is the leading cause of death in HIV-infected individuals owing to the high incidence of dual infections and the accompanying inhibition of the immune system by both HIV/ AIDS and TB. Ninety percent of the estimated deaths from TB and $95 \%$ of the estimated eight million new cases of TB each year occur in developing countries, which comprise $85 \%$ of the world's population ${ }^{2}$. Incidence is increasing fastest in African countries that are affected by HIV, followed by Eastern Europe and the former Soviet Union, both of which are plagued by multi-drug resistant strains of $\mathrm{TB}^{3,4}$ that present an ominous global threat.

The TB pandemic has continued despite widespread use of the only available licensed TB vaccine bacille Calmette-Guérin (BCG) ${ }^{5}$ under the WHO expanded program on immunization (EPI) and despite the use of directly observed chemotherapy programmes (DOTS) for those diagnosed with active disease. Failure or delay in diagnosis of active TB cases, relapse rates of up to $5 \%$ after DOTS ${ }^{6,7}$, and the lengthy treatment required to cure $\mathrm{TB}$, which often results in poor compliance and the consequent emergence of multi-drug resistant $M$. tuberculosis strains ${ }^{8-10}$, present serious challenges for the DOTS strategy. Although BCG seems to provide protection against disseminated disease in newborns and children, its efficacy against pulmonary TB is poor, which highlights the need for a better vaccine regimen.

There are two potential vaccination strategies against TB; those given before (pre-exposure, prophylactic) or after (postexposure, therapeutic) exposure to the pathogen. Therapeutic vaccines can also be used in combination with drug therapy. Specific vaccines designed to prevent latent $\mathrm{TB}$ or reactivation from the latent state are in the early stages of development. Here, we review the development of crucial new prophylactic vaccines, the primary goal of which is to prevent infection itself or disease that occurs after infection. Such vaccines include recombinant BCG (rBCG), attenuated strains of $M$. tuberculosis, subunit vaccine approaches and live, non-replicating viral vector-based delivery systems used alone or in prime-boost regimens. A formidable challenge facing the development of these new vaccines is their safety, especially in populations afflicted with both HIV and TB.

\section{Infection with Mycobacterium tuberculosis} The development of a delayed-type hypersensitivity response to tuberculin antigen (TST) is considered an indicator of $M$. tuberculosis infection. Only 20-50\% of individuals that are exposed to M. tuberculosis become infected, as evidenced by a positive TST in single case contact studies ${ }^{11}$. Around 5\% of infected individuals develop pulmonary disease or some other clinical form of TB over 2-5 years, whereas the other $95 \%$ of infected TST positive individuals develop latent TB infection (LTBI). Individuals with LTBI have about a 5\% lifetime chance of reactivating their latent infection, resulting in the development of clinical $\mathrm{TB}^{11}$.

M. tuberculosis is transmitted by the aerosol route of infection. After phagocytosis, the organism successfully hides from immune-mediated destruction inside the endosome of the macrophage using various mechanisms, which include changing the endosomal $\mathrm{pH}$, inhibiting apoptosis and destroying toxic superoxides that are secreted by immune cells. Infected alveolar macrophages and dendritic cells migrate to adjacent lymph nodes where mycobacterial antigens are presented and a T-helper-1 $\left(\mathrm{T}_{\mathrm{H}} 1\right)$-type response is initiated. This complex immune response ultimately results in the formation of the granuloma around infected macrophages. IFN- $\gamma$, which synergizes with tumour-necrosis factor- $\alpha$ (TNF- $\alpha$ ), is central to the activation of macrophages and the isolation of $M$. tuberculosis inside the granuloma. The granuloma is initially composed of $\mathrm{CD}^{+}$and $\mathrm{CD} 8^{+} \mathrm{T}$ cells, but a complex array of T cells, including $\mathrm{CD}^{+}, \mathrm{CD}^{+}, \gamma \delta \mathrm{T}$ cells and CD1-restricted $\alpha \beta$ T cells are also invoked to orchestrate immune responses and to contain the infection $^{12-14}$. At later stages, the granuloma is surrounded by a fibrotic wall and lymphoid follicular structures. The granuloma can persist for decades and can contain the tubercle infection in a state of dormancy by depriving mycobacteria of oxygen and nutrients.

However, failure to contain the infection can result in release of organisms, and caseation and necrosis with active clinical disease and transmission (FIG. 1).

Requirements for TB vaccine development Scientific rationale. Most humans have complete or partial resistance to tuberculous disease following exposure or infection with $M$. tuberculosis. Further indication that resistance to $\mathrm{TB}$ is mediated in part by the 
a
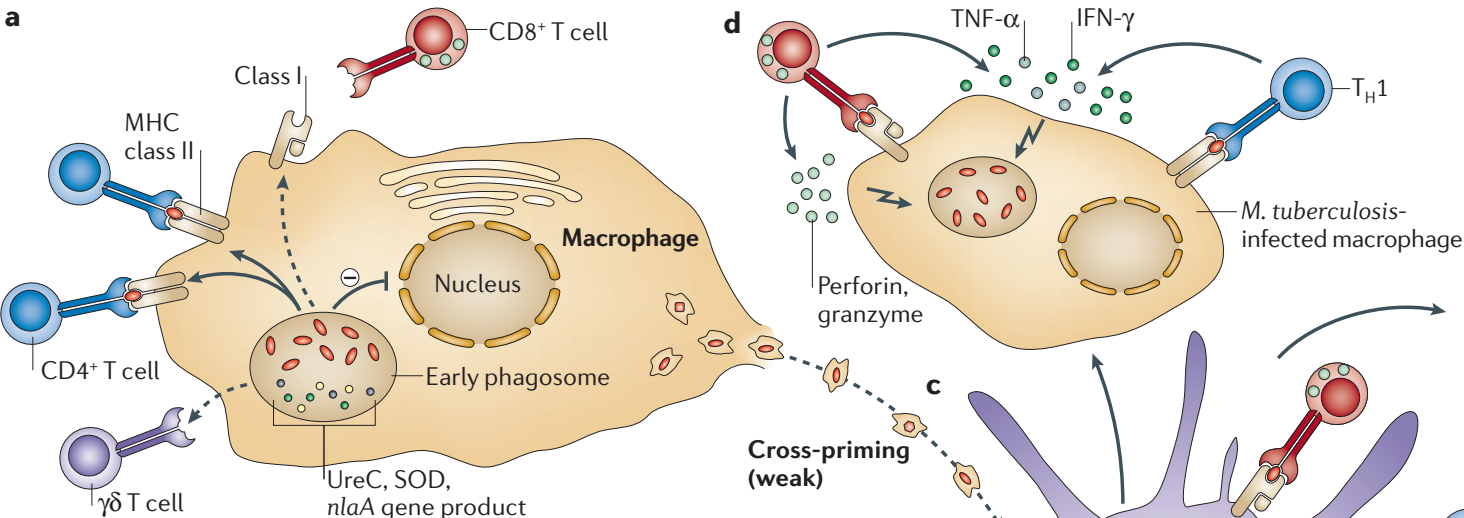

b
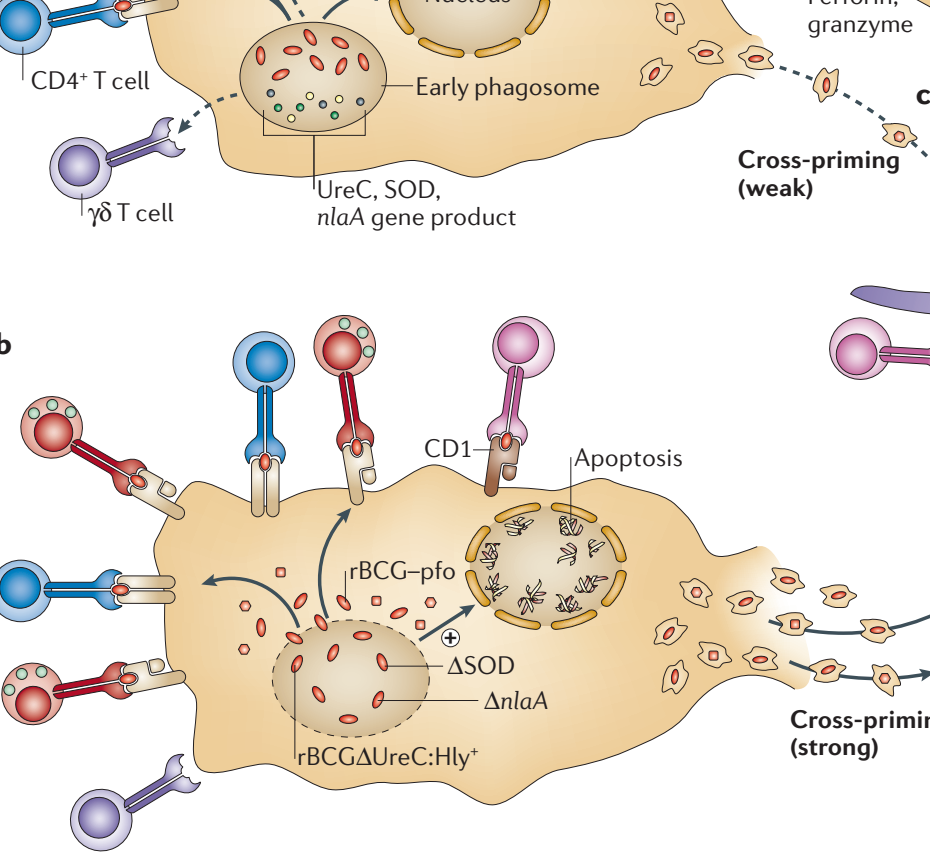

(⿻)

Cross-priming

(weak)
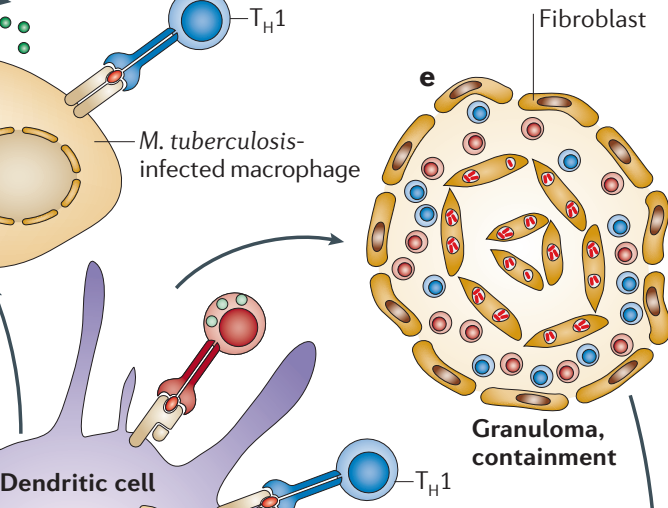

Dendritic cell
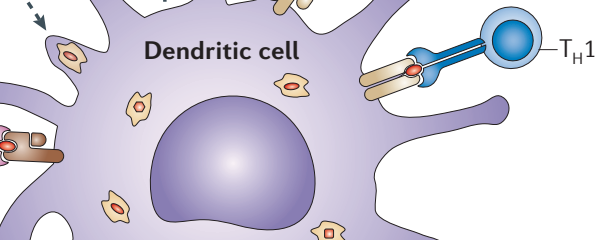

Figure 1 | The immune response to Mycobacterium tuberculosis infection or vaccination with BCG or recombinant modified BCG. a|M. tuberculosis subverts macrophage phagosome maturation through the production of UreC, which inhibits the acidification of the early phagosome. M. tuberculosis also produces factors such as superoxide dismutase (SOD) and the nIA gene product, which might inhibit host defences by interfering with host cell apoptosis. In the phagosome, M. tuberculosis antigens have access to the $\mathrm{MHC}$ class II machinery resulting in a predominant $\mathrm{CD} 4^{+} \mathrm{T}$-cell response. However, apoptosis of infected macrophages produces extracellular vesicles carrying glycolipids and protein antigens. These vesicles are taken up by dendritic cells as a result of cross-priming and presented in the context of MHC class I (CD $8^{+} \mathrm{T}$-cell responses), MHC class II (CD4 ${ }^{+} \mathrm{T}$-cell responses) and CD1 molecules. $\mathbf{b}$ | Enhancement of MHC class I antigen presentation and $\mathrm{CD} 8^{+} \mathrm{T}$-cell responses by modified $\mathrm{BCG}$ constructs. $\mathrm{rCC}$ vaccines such as rBCG-pfo (with deletion of UreC and expression of the $\mathrm{pH}$-independent perfringolysin) or rBCG $\Delta$ UreC:Hly ${ }^{+}$(with deletion of UreC and expression of the acidic $\mathrm{pH}$-dependent $\mathrm{Hly}^{+}$(listeriolysin)) facilitate the formation of pores in the membrane of the early phagosome, thereby allowing leakage of BCG mycobacteria and antigens into the cytoplasm. This leads to potent MHC class I presentation, enhancement of apoptosis and increased cross-priming and uptake by dendritic cells (c), resulting in enhanced $\mathrm{CD} 8^{+}$and $\mathrm{CD} 4^{+} \mathrm{T}$-cell responses as well as $\gamma \delta \mathrm{T}$-cell and CD1-restricted $\alpha \beta$ T-cell activation. rBCG constructs with diminished SOD activity or deletion of nlaA can further enhance apoptosis. $\mathbf{d} \mid \mathrm{CD} 8^{+} \mathrm{T}$ cells produce effector cytokines (IFN- $\gamma$ and TNF- $\alpha$ ) and lyse infected cells through the release of granules (perforin and granzyme) or by Fas-mediated lysis, whereas activated $\mathrm{CD}^{+} \mathrm{T}_{H} 1$ cells produce IFN- $\gamma$, which activates macrophages to kill M. tuberculosis. e | Infected macrophages can also be contained within granulomas in a state of mycobacterial dormancy for decades. $\mathbf{f} \mid$ However, M. tuberculosis can reactivate as a result of a weakened immune system, with release of organisms from the granuloma and progression to active clinical disease. immune system stems from the findings that certain groups of immunocompromised people have a high susceptibility to tuberculous disease. These include people with Mendelian inherited defects in immune mediators such as INF- $\gamma^{15,16}$, patients treated with TNF- $\alpha$-antagonist drugs for rheumatoid arthritis ${ }^{17}$, and AIDS patients with decreased numbers of $\mathrm{CD} 4^{+} \mathrm{T}$ cells that are also functionally impaired ${ }^{18}$. Results from clinical trials with BCG, although not consistent, have nonetheless provided proofof-principle that vaccination can be effective against the consequences of infection with M. tuberculosis. Therefore, although resistance to TB is in part a property of the hostmediated immune response, in people not naturally resistant, vaccination could confer an additional degree of protection.

Although animal-challenge models have not been proven to correlate with protection in humans, new rBCG vaccines or heterologous prime-boost regimens (TABLE 1) show better protection than BCG in such models. Such prime-boost regimens consist of a prime vaccine using $\mathrm{BCG}$ or $\mathrm{rBCG}$, followed by a heterologous booster vaccine that contains antigens shared with the prime.

\section{Immunological correlates of protection.}

Rapid vaccine development and improvement requires reliable correlates of vaccineinduced human protection. In mouse TB challenge studies, IFN- $\gamma$-secreting $\mathrm{CD} 4^{+}$ $\mathrm{T}$ cells are important mediators of protection $^{19,20}$, and attempts to induce TB-antigenspecific IFN- $\gamma$-secreting $\mathrm{CD} 4^{+} \mathrm{T}$ cells has been the dominant theme of most TB vaccine research. 
Table 1 |Summary of new-generation tuberculosis vaccine work in progress

\begin{tabular}{|c|c|c|c|}
\hline Vaccine & Description & Development stage & Researchers/sponsors \\
\hline \multicolumn{4}{|c|}{ Live mycobacterial vaccines: modified BCG } \\
\hline rBCG30 & $\begin{array}{l}\text { BCG Tice engineered to overexpress } \\
\text { Ag85B }\end{array}$ & $\begin{array}{l}\text { Phase I trial in US, completed in 2004; no } \\
\text { serious adverse events }\end{array}$ & $\begin{array}{l}\text { M. Horwitz, UCLA; D. Hoft, St Louis } \\
\text { University, US; T. Littlejohn, Winston, } \\
\text { Salem, North Carolina, US } \\
\text { Sponsor: Sequella, Aeras* }\end{array}$ \\
\hline rBCG-Aeras 403 & $\begin{array}{l}\text { BCG Danish with endosome escape } \\
\text { and overexpression of several proteins } \\
\text { including Ag85A, Ag85B and TB10.4 }\end{array}$ & $\begin{array}{l}\text { Ongoing pre-clinical studies and GMP } \\
\text { production; Phase I clinical trial planned } \\
\text { for } 2006\end{array}$ & $\begin{array}{l}\text { R. Sun, D. Hone, M. Stone, J. Sadoff, } \\
\text { Aeras* }\end{array}$ \\
\hline rBCG $\Delta$ Ure:CHly ${ }^{+}$ & BCG Pasteur with endosome escape & $\begin{array}{l}\text { Ongoing pre-clinical studies and GMP } \\
\text { production; Phase I clinical trial planned } \\
\text { for } 2006\end{array}$ & $\begin{array}{l}\text { S. Kaufmann, Max Planck Institute for } \\
\text { Infection Biology, Berlin, Germany }\end{array}$ \\
\hline BCG::RDI & $\begin{array}{l}\text { BCG Pasteur with reintroduction of RD-1 } \\
\text { locus which contains protective Ags }\end{array}$ & Ongoing pre-clinical studies & S. Cole, Institute Pasteur, Paris, France \\
\hline Pro-apoptotic BCG & $\begin{array}{l}\text { BCG Tice with diminished superoxide } \\
\text { dismutase activity }\end{array}$ & Ongoing pre-clinical studies & $\begin{array}{l}\text { D. Kernodle, Vanderbilt, US } \\
\text { Sponsor: NIH, VA, Aeras* }\end{array}$ \\
\hline $\begin{array}{l}\text { M. tuberculosis } \\
\mathrm{mc}^{2} 6030\end{array}$ & $\begin{array}{l}\text { Mtb H37Rv with deletion of panCD and } \\
\text { RD-1 locus }\end{array}$ & $\begin{array}{l}\text { Ongoing pre-clinical studies and GMP } \\
\text { production; Phase I clinical testing } \\
\text { planned for } 2006\end{array}$ & $\begin{array}{l}\text { W. Jacobs, Albert Einstein College of } \\
\text { Medicine, New York } \\
\text { Sponsor: NIH }\end{array}$ \\
\hline $\begin{array}{l}\text { M. tuberculosis } \\
\mathrm{mc}^{2} 6020\end{array}$ & $\begin{array}{l}\text { Mtb H37Rv with deletion of the lys } A \text { and } \\
\text { the panCD locus }\end{array}$ & $\begin{array}{l}\text { Ongoing pre-clinical studies and GMP } \\
\text { production; Phase I clinical testing } \\
\text { planned for } 2006\end{array}$ & $\begin{array}{l}\text { W. Jacobs, Albert Einstein College of } \\
\text { Medicine } \\
\text { Sponsor: NIH }\end{array}$ \\
\hline \multicolumn{4}{|l|}{ Subunit vaccines } \\
\hline Mtb72F & $\begin{array}{l}\text { Recombinant fusion protein (Mtb39 and } \\
\text { Mtb32) in AS02A and AS01B adjuvants }\end{array}$ & $\begin{array}{l}\text { Phase I trials completed in US and } \\
\text { Europe; no serious adverse events; } \\
\text { additional trials ongoing in Europe }\end{array}$ & $\begin{array}{l}\text { Y. Skeiky, S. Reed, Corixa Corp. Seattle, } \\
\text { US } \\
\text { Sponsor: Glaxo Smith Kline, Aeras* }\end{array}$ \\
\hline \multicolumn{4}{|c|}{ Naked DNA and viral-vectored vaccines } \\
\hline Hsp65 (GroEL) DNA & $\begin{array}{l}\text { Conserved antigen from Mycobacterium } \\
\text { leprae for immunotherapy }\end{array}$ & Ongoing pre-clinical studies & $\begin{array}{l}\text { D. Lowrie, National Institute for Medical } \\
\text { Research, London UK. } \\
\text { Sponsor: Sequella Inc. }\end{array}$ \\
\hline MVA85A & $\begin{array}{l}\text { Attenuated strain of vaccinia (modified } \\
\text { vaccinia virus) expressing Ag85A }\end{array}$ & $\begin{array}{l}\text { Completed and ongoing Phase I clinical } \\
\text { trials; immunogenic, no serious adverse } \\
\text { events reported }\end{array}$ & $\begin{array}{l}\text { A. Hill, H. McShane, Oxford University, } \\
\text { UK } \\
\text { Sponsor: Wellcome Trust }\end{array}$ \\
\hline $\begin{array}{l}\text { Aeras } 402 \\
\text { (Ad35.TB-S) }\end{array}$ & $\begin{array}{l}\text { Non-replicating Ad } 35 \text { expressing } \\
\text { multiple TB proteins including Ag85A, } \\
\text { Ag85B and TB10.4 }\end{array}$ & $\begin{array}{l}\text { Ongoing pre-clinical studies; GMP } \\
\text { production for Phase I clinical trials } \\
\text { planned for } 2006\end{array}$ & $\begin{array}{l}\text { Aeras }{ }^{\star} \text {, Crucell } \\
\text { Sponsor: Bill and Melinda Gates } \\
\text { Foundation }\end{array}$ \\
\hline \multicolumn{4}{|c|}{ Double-stranded RNA capsids } \\
\hline $\begin{array}{l}\text { Double-stranded } \\
\text { RNA capsids }\end{array}$ & $\begin{array}{l}\text { Double-stranded RNA capsids encoding } \\
\text { TB antigens for oral delivery }\end{array}$ & Ongoing pre-clinical studies & $\begin{array}{l}\text { J. Fulkerson, D. Hone, M. Stone, } \\
\text { D. Onyabe, J. Sadoff } \\
\text { Sponsor: Aeras* }\end{array}$ \\
\hline
\end{tabular}

*Supported by the Bill and Melinda Gates Foundation. Phase I clinical trial: trial that assesses the safety and immunogenicity of a drug or a vaccine in healthy volunteers. BCG, bacille Calmette-Guérin; GLP, good laboratory practice; GMP, good manufacturing practice; NIH, National Institutes of Health; SSI, Statens Serum Institute; TB, tuberculosis; VA, Medical Research Service, Department of Veterans Affairs.

MHC class I restricted, IFN- $\gamma$-secreting $\mathrm{CD}^{+} \mathrm{T}$ cells are also important in $\mathrm{TB}$ challenge models ${ }^{19,21}$ and experiments in knockout mice have reported that vaccines can induce significant protection in the absence of $\mathrm{CD}^{+} \mathrm{T}$ cells ${ }^{22}$. CD8 ${ }^{+} \mathrm{T}$ cells might also be important in the control of $\mathrm{LTBI}^{23}$

The potential role of both vaccineinduced $\mathrm{CD}^{+} \mathrm{T}$ cells and $\mathrm{CD} 8^{+} \mathrm{T}$ cells is not surprising as the granuloma is composed of $\mathrm{CD} 4^{+} \mathrm{T}$ cells with an outer ring of $\mathrm{CD}^{+} \mathrm{T}$ cells ${ }^{24,25}$. Potent new TB vaccines will probably need to induce a balanced antigen-specific $\mathrm{CD} 4^{+}$and $\mathrm{CD} 8^{+} \mathrm{T}$-cell 
response, but the detailed phenotypes and numbers of such cells required for protection is unknown. Furthermore, antibody production against mycobacterial cell wall components might also be important ${ }^{26}$.

At present, there are no proven human immunological correlates of vaccine-induced protection. The detailed phenotypic properties and levels of vaccine-induced $\mathrm{T}$ cells in protected versus non-protected volunteers in Phase IIb and Phase III efficacy studies are needed to generate such correlates. In an ongoing trial conducted by the South African TB Vaccine Initiative (SATVI) group (G. Hussey, T. Hawkridge, L. Geiter and W. Hanekom), cells from over 7,000 BCG-vaccinated 10-week-old infants have been collected and banked ${ }^{27,105}$ for the detailed analysis of vaccine-induced T cells. One correlate of protective immunity that could be explored using this approach is the activation of antigen-specific memory $\mathrm{CD}^{+}$and $\mathrm{CD}^{+} \mathrm{T}$ cells that secrete IFN- $\gamma$, IL-2 and TNF- $\alpha^{28}$. Combinations of these and other phenotypic markers in individual T cells - such as CD69, MIP1 $\beta$, CD45RA, CD27, memory markers such as CD127 (IL-7 receptor $^{28,29}$, cytotoxic markers such as FAS ligand, CD107a/b and CD3/4/8 phenotype $e^{28}$ - show potential as correlates of immune protection. $\gamma \delta \mathrm{T}$ cells that recognize phospholigands, and CD1-restricted T cells that recognize the abundant glycolipids of the mycobacterial cell wall through CD1 molecules (reviewed in REFS 12-14), might also require examination for their potential as immune correlates.

\section{Strategies to improve BCG}

The current BCG vaccine. BCG, the attenuated strain of Mycobacterium bovis, was derived from the parental strain by passage on potato-derived media. Almost three billion doses have been used in various forms since 1921. Approximately 115 million doses are distributed each year ${ }^{30,31}$ providing almost $80 \%$ coverage of infants worldwide ${ }^{32}$.

Neonatal vaccination with BCG has been reported as effective in reducing the incidence of childhood TB in endemic areas ${ }^{33}$ and in reducing the rate of complications from disseminated TB. However, the ongoing SATVI trial of over 11,000 newborns vaccinated with BCG showed an incidence of culture or smear-proven TB of around 3\% by the age of 3 years ${ }^{27,105}$.

The protective effect of BCG might wane over time resulting in a variable outcome that is insufficient to control TB in most endemic areas ${ }^{34}$. Although several older studies demonstrated efficacy as high as $80 \%$ in children, adolescents and young adults, with effects lasting up to 60 years in a study of native Americans ${ }^{35-37}$, and a recent study from Turkey claims that vaccination with two doses of BCG confers some protection in children against infection and disease ${ }^{38}$, a large-scale study from India over a wide age range did not show any overall efficacy of two different BCG vaccines compared with placebo $^{39}$.

The reasons for the varying efficacy of BCG in protection against pulmonary TB are not completely understood. Potential explanations that have been suggested (reviewed in REFS 30,32) include: interference with the immune response to BCG caused by previous exposure to environmental mycobacteria; differences among BCG vaccine sub-strains; and phenotypic changes in the vaccine during passage from the original cultures and during the manufacturing process; the deletion of protective antigens from BCG; failure of BCG to stimulate adequate, balanced anti-mycobacterial $\mathrm{CD} 4^{+}$ and $\mathrm{CD}^{+} \mathrm{T}$-cell responses; variability in dose, route of administration, age of administration and genetic differences among vaccinees; and lyophilization of the vaccine. Several strategies are being used to improve the efficacy of BCG and are described below.

\section{Antigen overexpression. Antigen 85B} (Ag85B) is a secretory and immunogenic protein of $M$. tuberculosis and BCG with mycolyl-transferase activity, which is required for mycobacterial cell wall synthesis. M. Horwitz's group (UCLA) have produced a TB vaccine candidate, rBCG30 that overexpresses Ag85B and that induces increased protection compared with its parental BCG strain in animal challenge studies $^{40,41}$. There were no significant safety issues in a Phase I clinical trial of rBCG30 in over 30 healthy adult volunteers. Increased immunogenicity compared with the parent BCG strain was reported ${ }^{42}$, although the study did not meet its predefined immunogenicity endpoints.

Endosome escape. Because protective immunity against $M$. tuberculosis infection seems to involve both $\mathrm{CD}^{+}$and $\mathrm{CD} 8^{+} \mathrm{T}$ cells, antigens must be presented by both MHC class I and II molecules. BCG that resides in the phagosome gains access to MHC class II molecules, thereby primarily activating $\mathrm{CD} 4^{+} \mathrm{T}$ cells. To be presented by MHC class I molecules and to activate $\mathrm{CD} 8^{+} \mathrm{T}$ cells, BCG must either escape from the endosome and gain access to the cytoplasm of the infected cells or induce apoptosis-mediated CD8 ${ }^{+} \mathrm{T}$-cell responses. Listeria monocytogenes Hly (listeriolysin) is a sulphydryl-activated cytolysin that forms pores in the membrane of the early phagosome. S. Kaufmann's group (Max Planck Institute) have produced an $\mathrm{rBCG}$ vaccine (rBCG $\Delta$ UreC:Hly ${ }^{+}$) that secretes Hly, which punches holes in the endosome and allows some leakage of BCG from the phagosome into the cytoplasm of infected host cells ${ }^{43}$, also resulting in apoptosis of these cells (FIG. 1). Because the activity of Hly is optimal in an acidic environment, the BCG urease gene (ureC), which enables mycobacteria to block the acidification of the early macrophage phagosome, was also deleted. Enhanced presence of BCG in the cytoplasm increases $\mathrm{MHC}$ class I presentation of BCG antigens to $\mathrm{CD}^{+} \mathrm{T}$ cells, thereby increasing the $\mathrm{CD} 8^{+}$ T-cell immune response. Furthermore, translocation of BCG into the cytoplasm triggers apoptosis, which in turn kills the bacilli and releases antigens, a powerful signal for induction of cellular immunity ${ }^{44}$. The antigens released as apoptotic blebs (vesicles that carry mycobacterial antigens) are subsequently taken up and processed by dendritic cells, which activate $\mathrm{T}$ cells by a mechanism referred to as 'cross-priming' (reviewed in REF. 45). In preclinical mouse-protection studies, the rBCG $\Delta$ UreC:Hly ${ }^{+}$vaccine induced better efficacy than the BCG strain and was safer in immunocompromised SCID mice ${ }^{46}$. Also, unlike the parental BCG strain the $\triangle \mathrm{UreC}: \mathrm{Hly}^{+} \mathrm{rBCG}$ was shown to protect significantly against the virulent $M$. tuberculosis strain, Beijing/W. This vaccine is scheduled for Phase I trials in early 2006 (REFS 47,48).

R. Sun, M. Stone and D. Hone's group at Aeras have constructed rBCG strains that escape from the endosome using the $\mathrm{pH}$-independent perfringolysin (rBCG-pfo, (FIG. 1)), instead of $\mathrm{Hly}^{49}$, with overexpression of several immunodominant antigens including Ag85A, Ag85B and TB10.4. This strain combines the increased protection seen with $\mathrm{rBCG} 30$ with the increased protection and safety of the endosome-escape mutants. This vaccine is scheduled for Phase I trials in 2006 (REF. 27).

Reducing BCG interference with immunity. Usually, host superoxides produced by macrophages kill $M$. tuberculosis and can amplify host T-cell-recruiting signals. D. Kernodle's group (Vanderbilt) has shown that secretion of superoxide dismutase (SOD) and other compounds that inactivate superoxides by M. tuberculosis promotes granuloma formation in which $M$. tuberculosis is secluded, and therefore contributes to inhibition of host defences and persistence of the organism ${ }^{50}$. 
Attenuated M. tuberculosis and rBCG with reduced secretion of functional SOD are more potent vaccines than their parental strains ${ }^{51}$.

M. tuberculosis and BCG, similar to other successful human pathogens, also produce various other gene products that inhibit the human immune system. W. Jacobs (Albert Einstein College of Medicine) recently suggested that promoting a pro-apoptotic vaccine by specific deletion of genes such as nlaA (Rv3238c), which inhibit apoptosis of infected cells, might also lead to a superior vaccine ${ }^{52}$. Cells infected with rBCG or recombinant M. tuberculosis vaccines that cannot inhibit caspase-8-mediated apoptosis that usually accompanies infection consequently undergo apoptosis. This leads to cross priming and powerful cellular immune responses against these live vaccines, as caspase-8-mediated apoptosis is one of the most powerful known inducers of cellular immunity ${ }^{53,54}$.

\section{Reintroduction of deleted genes. S. Cole's} group (Institute Pasteur) have reintroduced selected genes into BCG that were deleted during BCG attenuation ${ }^{55}$. Examples include the RD1 locus ${ }^{56}$ (see TubercuList in Online links box), which encodes the immunodominant and protective ESAT- 6 and CFP-10 proteins - reintroduction of this locus enhanced the protective efficacy of the recombinant BCG::RD1-2F9 vaccine compared with $\mathrm{BCG}^{57}$. However, this approach, although increasing the potency of BCG, might also increase its virulence ${ }^{58}$ and pose problems for human use.

\section{New attenuated live TB vaccines}

An alternative to BCG modification is to use molecularly attenuated $M$. tuberculosis that contains over $120 \mathrm{M}$. tuberculosis genes not found in $\mathrm{BCG}^{59-61}$. A desirable feature of effective and safe live $M$. tuberculosis vaccine strains is their ability to persist in the host for a limited duration, greater protective efficacy, as well as an improved safety profile compared with the currently used BCG vaccine. The obstacles facing live TB vaccines with respect to safety concerns, public perception and regulatory hurdles were discussed at a recent meeting, and the danger of reversion of live TB vaccines to a virulent state was considered a crucial issue ${ }^{62}$. At the minimum, two non-reverting independent mutations as well as proven safety in immunocompromised SCID mice are recommended for attenuated M. tuberculosis vaccines. Also, the potential release of antibiotic-resistance gene markers from live vaccines into the environment, with subsequent transfer to other organisms, is considered an important concern.
Inactivation of PhoP, which regulates expression of many $M$. tuberculosis proteins, by C. Soto's group (University of Zaragoza, Spain) ${ }^{63}$ attenuated $M$. tuberculosis virulence while maintaining the pattern of immune responses associated with the parental $M$. tuberculosis strain ${ }^{64}$. This mutant has shown promising early animal protection but will probably require a second, independent, nonreverting mutation to satisfy human safety concerns ${ }^{62}$.

Auxotrophic mutant vaccine candidates (requiring the addition of a nutrient(s) for survival) have been produced by transposon mutagenesis. These mutants maintain their infective ability, have a limited duration of replication in the host ${ }^{65,66}$ and protect against virulent $\mathrm{TB}$ challenge in the guinea pig model ${ }^{67}$. The extent of the attenuation and safety of these vaccines remains to be defined in immunocompromised SCID mice. The Jacobs and Bloom groups have targeted the deletion of proteins involved in M. tuberculosis lipid metabolism by disrupting both the panC and panD genes (which are involved in pantothenic acid synthesis). Further deletion of the lys $A$ gene produced vaccine $\mathrm{mc}^{2} 6020$ (panC panD lys $A$ ), which does not replicate in vivo. Deletion of the $\mathrm{RD} 1$ region (implicated in the attenuation of BCG) from the panC, panD mutant produced vaccine $\mathrm{mc}^{2} 6030$ (panC panD $\triangle \mathrm{RD} 1)^{68-70}$. Both of these highly attenuated mutants were safer than BCG in immunocompromised animals and provided similar levels of protection in mouse challenge studies. The $\mathrm{mc}^{2} 6030$ is scheduled for Phase I trials in 2006 (REF. 52).

\section{Subunit vaccine strategies}

Vaccine antigen identification. Antigens of M. tuberculosis that activate T cells in previously infected humans and animals are considered good vaccine candidates. Biochemical approaches initially identified several of the most abundant proteins (Ag85 complex, MPT32, PhoS, DnaK, GroES, MPT46, MPT53, MPT63 and the 19-kDa lipoprotein) secreted by $M$. tuberculosis ${ }^{71-73}$. Subsequently, antigens in the low molecular mass range of 6-10 kDa (ESAT-6 family) and 26-34 kDa (Ag85B and MPT59) ${ }^{74,75}$ were defined. T-cell and serological expression cloning approaches ${ }^{76-79}$ identified several important dominant immunogenic antigens including Mtb32 (a secreted serine protease) and Mtb39 (a member of the PPE (proline-prolineglumatic acid) family of proteins presumably localized on the cell wall ${ }^{80,81}$. Proteomic approaches ${ }^{82,83}$ have also identified several promising antigens such as Rv3407 (REF. 84).
Antigen vaccines have advanced towards clinical development using animal protection models and assessment of their potency in stimulating immune cells derived from peripheral blood mononuclear cells of healthy, previously infected donors to produce IFN- $\gamma$. Antigen vaccine candidates therfore include Ag85A and Ag85B (REFS 85,86), ESAT-6, TB10.4 and Mtb9.9 (REFS 75,79,87) and the PPE family of proteins, Mtb39a-e and Mtb41 (REFS 76,78,81).

Recombinant fusion proteins. Y. Skeiky, S. Reed and P. Andersen's groups have used a genetic fusion approach to generate hybrid protein vaccines consisting of Mtb39 and Mtb32 (Mtb72F) ${ }^{88}$, or Ag85B and ESAT-6 $(\text { Hybrid-1 })^{89}$, respectively. These candidate vaccines were selected on the basis of their strong recognition by immune cells in infected individuals and experimentally vaccinated or infected animals. The generation of recombinant protein subunit vaccines comprising multiple open reading frames is both cost effective and simple, and the fusion vaccines can be more immunogenic than the individual components. Both fusion recombinant vaccine constructs (Mtb72F and Hybrid-1) in selected adjuvants protect as well as BCG in the mouse and guinea pig TB challenge models ${ }^{88,90-92}$. Multiple Phase I clinical trials of Mtb72F have been completed in the United States and Europe (results pending) and additional trials are being conducted in Europe. Phase I trials of Hybrid-I started in Europe in November 2005.

ESAT-6 (the component of Hybrid-I that is absent from all BCG, but present in $M$. tuberculosis strains) is the basis of a new generation of diagnostic tests for $M$. tuberculosis infection. Therefore, if used as a vaccine, Hybrid-I could potentially compromise the diagnostic utility of ESAT- 6 in distinguishing between infection and vaccination $^{93,94}$. Therefore, P. Andersen's group in collaboration with Aeras has developed a new fusion construct, HyVac-4, which consists of Ag85B-TB10.4, and which avoids diagnostic interference and should also boost BCG-primed individuals because TB10.4 is immunogenic in BCG-vaccinated subjects $^{87,95}$.

Prime-boost vaccine strategies. The attraction of the heterologous prime-boost approach is that it preferentially expands TB-specific pre-existing memory T cells against antigenic epitopes shared by both the prime and booster vaccines. This approach also minimizes the potential of 
generating antibody and T-cell neutralizing effects that would impact on the 'take' and effectiveness of a non-replicating viralvector boost (FIG. 2).

An early study using a BCG prime-boost approach showed that mice boosted with an adjuvanted Ag85A protein when they had lost their BCG-induced capacity to resist an aerosol TB challenge were protected better than non-BCG-primed animals given adjuvanted protein ${ }^{96}$. This suggests that adjuvanted proteins including Mtb72F, Hybrid- 1 and HyVac- 4 could induce superior protection when given as boosters in primary regimens, or in adolescence.

Such recombinant protein regimens, however, might induce primarily antigen-specific $\mathrm{CD}^{+}{ }^{+} \mathrm{T}$ cells instead of the desired balanced $\mathrm{CD}^{+}$and $\mathrm{CD}^{+}{ }^{+} \mathrm{T}$-cell responses. A recent study in mice of a BCG prime followed by a booster with an $M$. tuberculosis DNA vaccine encoding the gene product Rv3407 (a 10-kDa protein of unknown function) also showed superior protection compared with BCG alone $^{84}$. Protection that was superior to BCG was also demonstrated in guinea pigs immunized with a BCG prime-Mtb72F DNA boost regimen followed by aerosol challenge with virulent $M$. tuberculosis. Remarkably, histological examination of the lungs of these guinea pigs revealed minimal granulomatous lesions with evidence of lesion healing and airway remodelling at a time when animals immunized with BCG and then challenged were dying ${ }^{90}$.

Whereas, so far, the success of human DNA vaccination in general has been disappointing, non-replicating recombinant poxviruses and adenoviruses are efficient at boosting previously primed $\mathrm{T}$-cell responses and show great potential for use in heterologous prime-boost immunization strategies. Priming with BCG and boosting with a recombinant Modified Vaccinia Ankara virus (MVA) that expresses Ag85A induced increased levels of $\mathrm{CD} 4^{+}$and $\mathrm{CD} 8^{+}$ $\mathrm{T}$ cells and enhanced protection in animals compared with a vaccination regimen using either BCG or MVA85A alone ${ }^{97}$. In a Phase I clinical trial recently reported by $\mathrm{H}$. McShane and A. Hill's group (Oxford University, UK), immunization of volunteers from 6 months to 38 years after BCG vaccination with MVA resulted in persistent, high levels of IFN- $\gamma$ secreting $\mathrm{CD}^{+} \mathrm{T}$ cells, which were substantially higher than seen following vaccination with BCG or MVA85A alone, which only induced transient responses ${ }^{106}$.

Adenoviral vectors are potent vaccinedelivery vehicles. A recent study showed that vaccination of mice by the intranasal

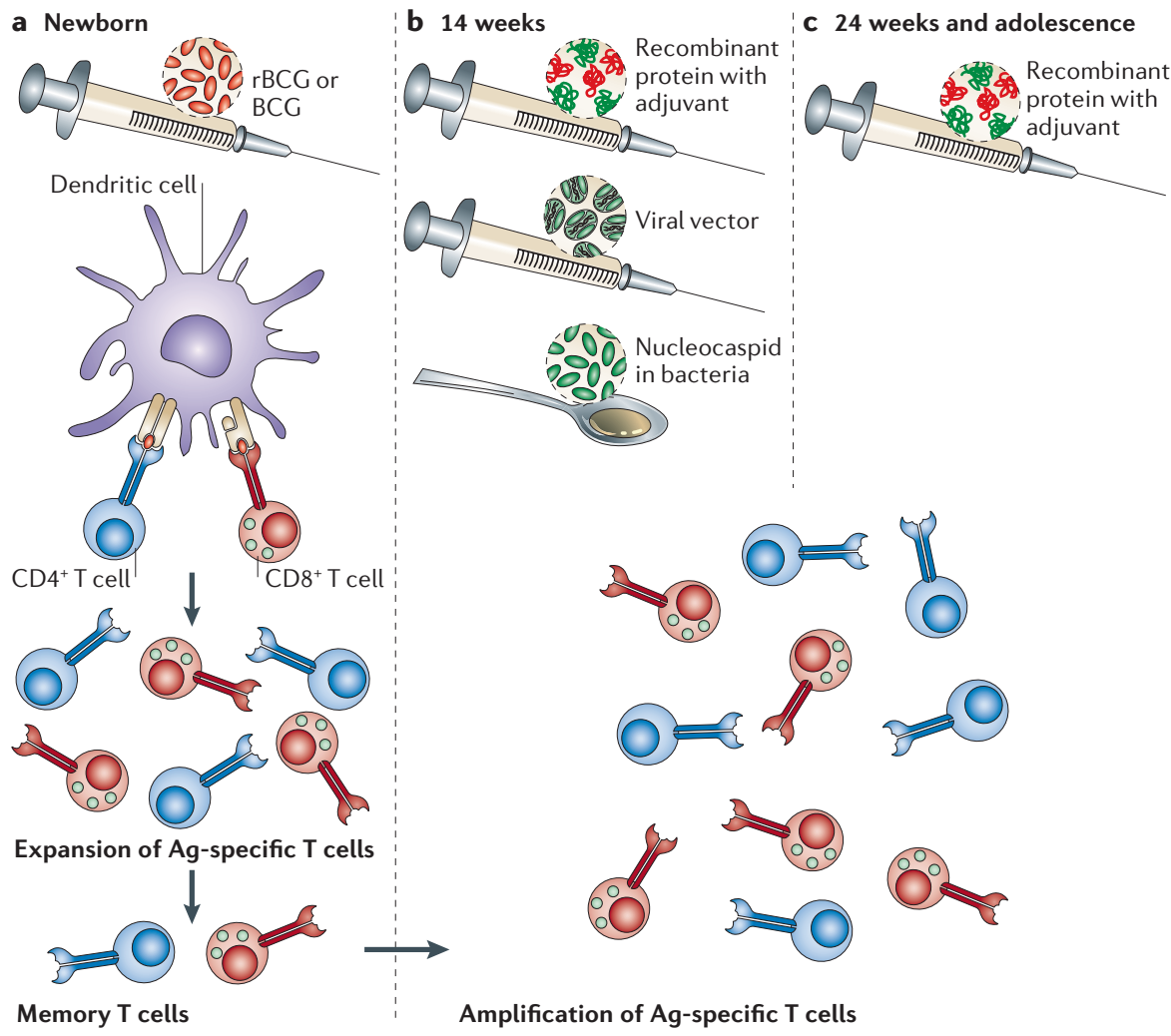

Figure 2 | Prime-boost vaccination strategies. Boosting or augmenting BCG or a recombinant, modified $\mathrm{BCG}(\mathrm{rBCG})$, rather than trying to completely replace it, is a practical approach for future immunization against TB. Heterologous prime-boost regimens begin with vaccination of newborns using BCG or modified rBCG as the prime (a). This is followed by a booster vaccination in infancy (b) and later in childhood and adolescence (c) using different antigen-delivery systems (heterologous boosting) composed of selected antigens in common with the prime. The booster can include viral vectors such as recombinant adenovirus or poxvirus engineered to express immunogenic and protective mycobacterial candidate antigens (for example, Ag85A, Ag85B and TB10.4) or recombinant proteins (for example, HyVac-4, Hybrid-1 and Mtb72F) delivered with adjuvants (such as AS02A, AS01B, IC31 and LipoVac). Priming with BCG or rBCG followed by a booster with adenovirus and a subsequent boost with recombinant protein in adjuvant formulation is also being contemplated. Alternatively, Shigella spp. nucleocapsid vectors offer a possible alternative oral vaccine boost (b). Shigella vectors deliver double stranded RNA capsids into the cytoplasm where they produce mRNA that directs cellular production of $M$. tuberculosis antigens. The heterologous prime-boost approach preferentially expands BCG or TB-specific pre-existing memory T cells against antigenic epitopes shared by both the prime and booster vaccines. Administration of the booster vaccination in an alternative vector minimizes the generation of anti-vector immunity that is typically seen with repeated administration using the same vaccine (homologous boosting), thereby inducing a powerful synergistic immune response. Unlike BCG (which induces a predominant $\mathrm{CD} 4^{+} \mathrm{T}$-cell response), $\mathrm{rBCG}$ that has access to the cytoplasm activates a broad repertoire of antigen-specific $\mathrm{CD}^{+}$and $\mathrm{CD} 8^{+} \mathrm{T}$ cells (a). Subsequent boosting results in the expansion of selected antigen-specific memory $\mathrm{CD} 4^{+}$and $\mathrm{CD} 8^{+} \mathrm{T}$ cells and the selective enrichment of high avidity $T$ cells to combat exposure to the pathogen $(\mathbf{b}, \mathbf{c})$.

mucosal route with a recombinant adenoviral (Ad5 based) construct expressing the $M$. tuberculosis antigen Ag85A (AdAg85A) conferred protection (mediated by both $\mathrm{CD}^{+}$and $\mathrm{CD}^{+} \mathrm{T}$ cells) that was superior to that seen with $B C G$ vaccination against a pulmonary $M$. tuberculosis challenge ${ }^{98}$. Aeras, in collaboration with Crucell, has constructed non-replicating Ad35 vectors that are less prevalent in the environment than Ad5 (REFS 99 and 100), and that express several
M. tuberculosis proteins including Ag85A, Ag85B and TB10.4 (REFS 27,100). Vaccination of mice with a single dose of the rAd35-TB construct conferred protection against $M$. tuberculosis challenge ${ }^{107}$. BCG prime followed by a booster with a single dose of these adenovirus recombinant vaccines in mice have yielded significantly increased antigenspecific $\mathrm{CD}^{+}$and $\mathrm{CD} 8^{+} \mathrm{T}$-cell responses compared with adenovectored $\mathrm{TB}$ vaccines or BCG administered alone. Aeras has 
constructed rBCG vaccines with over 50\% escape from the endosome that overexpress these same antigens to function as a prime for this adenovector boost ${ }^{27}$ (R. Sun et al., unpublished observations).

J. Fulkerson, D. Hone, D. Onyabe and M. Stone at Aeras have also constructed double stranded RNA capsids which can be orally delivered using engineered Shigella spp. vectors. Shigella delivers these capsids into the cytoplasm where they produce mRNA that directs cellular production of $M$. tuberculosis antigens $^{101}$ (J. Fulkerson et al., unpublished observations) and induces cellular immunity. Such an oral vaccine boost to an $\mathrm{rBCG}$ prime might have the low cost and ease of deliverability desirable in a TB vaccine for the developing world.

\section{Conclusions}

A safe and effective heterologous primeboost regimen, which boosts or augments $\mathrm{BCG}$ or $\mathrm{rBCG}$, is perhaps the most realistic strategy for future TB control through immunization. Ideally the $\mathrm{rBCG}$ prime in such a regimen would include the overexpression of important antigens from different stages of the pathogen's life cycle and would induce cross-priming and increased $\mathrm{CD} 4^{+}$and $\mathrm{CD} 8^{+}$ $\mathrm{T}$-cell responses, as well as increased safety in immunocompromised individuals. The ideal booster would comprise recombinant proteins delivered with adjuvants, or delivered by non-replicating viral or capsid vectors. Although there is a possibility that the current generation of new vaccines might protect against reactivation from the latent state, these vaccines are not generally focused on the 'latency' dosR regulated antigens recently identified by microarray and other techniques ${ }^{102,103}$. Furthermore, animal models of latency for preclinical vaccine evaluation are just being developed ${ }^{104}$. The best hope for these vaccines probably lies with overexpression of $d o s R$ regulated proteins by $\mathrm{rBCG}$ followed by a multivalent booster vaccine. Based on current clinical development timelines, first generation vaccine regimens for protection against infection and disease could be licensed and available in the next 7-9 years with vaccines against latency and reactivation 2-3 years later. Because of its great promise and practical usefulness, rBCG primeheterologous boost strategies for malaria and HIV are also currently being explored.

\footnotetext{
Yasir A. W. Skeiky and Jerald C. Sadoff are at the Aeras Global TB Vaccine Foundation, 1405 Research Blvd, Rockville, Maryland 20850, USA. e-mails: yskeiky@aeras.org; jsadoff@aeras.org doi10.1038/nrmicro 1419
}

1. Kochi, A. The global tuberculosis situation and the new control strategy of the World Health Organization 1991. Bull. World Health Organ.79, 71-75 (2001).

2. Hopewell, P. C. Impact of human immunodeficiency virus infection on the epidemiology, clinical features, management and control of tuberculosis. Clin. Infect. Dis. 15, 540-547 (1992).

3. Raviglione, M. C. et al. Tuberculosis trends in eastern Europe and the former USSR. Tuber. Lung Dis. 75, 400-416 (1994).

4. Maher, D. et al. Tuberculosis deaths in countries with high HIV prevalence: what is their use as an indicator in tuberculosis programme monitoring and epidemiological surveillance? Int. J. Tuberc. Lung Dis. 9, 123-127 (2005)

5. Bloom, B. R. \& Fine, P. E. In Tuberculosis: Pathogenesis, Protection, and Control (ed. Bloom, B. R.) 531-557 (American Society for Microbiology, Washington DC, 1994).

6. Summary from the 6th Annual Conference of the International Union Against Tuberculosis and Lung Disease. (Chicago, 2001)

7. Ormerod, L. P., Horsfield, N. \& Green, R. M Tuberculosis treatment outcome monitoring Blackburn 1988-2000. Int. J. Tuberc. Lung Dis. 6 662-665 (2002)

8. Sudre, P., ten Dam G. \& Kochi, A. Tuberculosis: a global overview of the situation today. Bull. World Health Organ.70, 149-159 (1992)

9. Dolin, P. J., Raviglione, M. C. \& Kochi, A. Global tuberculosis incidence and mortality during 19902000. Bull. World Health Organ. 72, 213-220 (1994).

10. Hopewell, P. et al. Evaluation of new anti-infective drugs for the treatment and prevention of infections caused by the Mycobacterium avium complex. Infectious Diseases Society of America and the Food and Drug Administration. Clin Infect. Dis. 15 (Suppl. 1), 296-306 (1992)

11. Smith, P. G. \& Moss, A. R. In Tuberculosis: Pathogenesis, Protection, and Control. (ed Bloom, B. R.) 47-59 (American Society for Microbiology, Washington DC, 1994)

12. Kaufmann, S. H. $\&$ McMichael, A. J. Annulling a dangerous liaison: vaccination strategies against AIDS and tuberculosis. Nature Med. 11 (Suppl. 4), 33-44 (2005)

13. Lewinsohn, D. M., Lewinsohn, D. A. \& Grotzke, J. E. TB vaccines at the turn of the century: insights into immunity to $M$. tuberculosis and modern approaches for prevention of an ancient disease. Semin. Respir Infect. 18, 320-338 (2003).

14. Flynn, J. L. \& Chan, J. Immunology of tuberculosis. Annu. Rev. Immunol. 19, 93-129 (2001).

15. Jouanguy, E. et al. Interferon- $\gamma$-receptor deficiency in an infant with fatal bacille Calmette-Guérin infection. N. Engl. J. Med. 335, 1956-1961 (1996).

16. Jouanguy, E. et al. A human IFNGR1 small deletion hotspot associated with dominant susceptibility to mycobacterial infection. Nature Genet. 21, 370-378 (1999).

17. Toussirot, E. \& Wendling, D. The use of TNF- $\alpha$ blocking agents in rheumatoid arthritis: an overview. Expert Opin. Pharmacother. 5, 581-594 (2004)

18. Havlir, D. V. \& Barnes, P. F. Tuberculosis in patients with human immunodeficiency virus infection. N. Engl. J. Med. 340, 367-373 (1999).

19. Cooper, A. M. et al. Disseminated tuberculosis in IFN- $\gamma$ gene-disrupted mice. J. Exp. Med.178, 2243-2247 (1993)

20. Flynn, J. L. et al. An essential role for IFN- $\gamma$ in resistance to Mycobacterium tuberculosis infection. J. Exp. Med. 178, 2249-2254 (1993).

21. Flynn, J. L. et al. Major histocompatibility complex class I-restricted T cells are required for resistance to Mycobacterium tuberculosis infection. Proc. Natl Acad. Sci. USA 89, 12013-12017 (1992).

22. Derrick, S. C. et al. Immunization with a DNA vaccine cocktail protects mice lacking CD 4 cells against an aerogenic infection with Mycobacterium tuberculosis. Infect. Immun. 72, 1685-1692 (2004).

23. van Pinxteren, L. A. et al. Control of latent Mycobacterium tuberculosis infection is dependent on CD8 T cells. Eur. J. Immunol. 30, 3689-3698 (2000).

24. Gonzalez-Juarrero, M. et al. Temporal and spatial arrangement of lymphocytes within lung granulomas induced by aerosol infection with Mycobacterium tuberculosis. Infect. Immun. 69, 1722-1728 (2001).
25. Ulrichs, T. et al. Human tuberculous granulomas induce peripheral lymphoid follicle-like structures to orchestrate local host defence in the lung. J. Pathol. 204, 217-228 (2004).

26. Teitelbaum, R. et al. A mAb recognizing a surface antigen of Mycobacterium tuberculosis enhances host survival. Proc. Natl Acad. Sci. USA 95 , 15688-15693 (1998).

27. Sadoff, J. Public private partnership approach to vaccine development. Presented at New Approaches to Vaccine Development. (Sept. 8-10, Berlin, Germany, 2005).

28. Bouneaud, C. et al. Lineage relationships, homeostasis, and recall capacities of central- and effector-memory CD8 T cells in vivo. J. Exp. Med. 201 579-590 (2005).

29. Seder, R. A. \& Ahmed, R. Similarities and differences in $\mathrm{CD}^{+}$and $\mathrm{CD}^{+}$effector and memory $\mathrm{T}$ cell generation. Nature Immunol. 4, 835-842 (2003).

30. Brewer, T. F. \& Colditz, G. A. Relationship between bacille Calmette-Guérin (BCG) strains and the efficacy of BCG vaccine in the prevention of tuberculosis. Clin. Infect. Dis. 20, 126-135 (1995).

31. Colditz, G. A. et al. Efficacy of BCG vaccine in the prevention of tuberculosis. Meta-analysis of the published literature. JAMA 271, 698-702 (1994).

32. Fine, P. E. M., Carneiro, I. A. M., Milstien, J. B. \& Clements, C. J. Issues relating to the use of BCG in immunization programmes (World Health Organization, Geneva, 1999).

33. Colditz, G. A. et al. The efficacy of bacillus CalmetteGuerin vaccination of newborns and infants in the prevention of tuberculosis: meta-analyses of the published literature. Pediatrics 96, 29-35 (1995).

34. Sterne, J. A., Rodrigues, L. C. \& Guedes, I. N. Does the efficacy of BCG decline with time since vaccination? Int. J. Tuberc. Lung Dis. 2, 200-207 (1998).

35. Aronson, N. E. et al. Long-term efficacy of BCG vaccine in American Indians and Alaska Natives: A 60-year follow-up study. JAMA 291, 2086-2091 (2004).

36. Fine, $P$. E. The BCG story: lessons from the past and implications for the future. Rev. Infect. Dis. 11 (Suppl. 2), 353-359 (1989).

37. Comstock, G. W. Field trials of tuberculosis vaccines: how could we have done them better? Control Clin. Trials 15, 247-276 (1994).

38. Soysal, A. et al. Effect of BCG vaccination on risk of Mycobacterium tuberculosis infection in children with household tuberculosis contact: a prospective community-based study. Lancet 366, 1443-1451 (2005).

39. Tuberculosis Research Centre (ICMR), Chennai. Fifteen year follow up of trial of BCG vaccines in south India for tuberculosis prevention. Indian J. Med. Res. 110, 56-69 (1999).

40. Horwitz, M. A. \& Harth, G. A new vaccine against tuberculosis affords greater survival after challenge than the current vaccine in the guinea pig model of pulmonary tuberculosis. Infect. Immun. 71 1672-1679 (2003).

41. Horwitz, M. A. et al. Recombinant bacillus CalmetteGuérin (BCG) vaccines expressing the Mycobacterium tuberculosis 30-kDa major secretory protein induce greater protective immunity against tuberculosis than conventional BCG vaccines in a highly susceptible animal model. Proc. Natl Acad. Sci. USA 97, 13853-13858 (2000).

42. Hoft, D. F. Results of the 1 st Phase I trial of a recombinant BCG TB vaccine ( $\mathrm{rBCG} 30)$. Presented at US-Japan Cooperative Medical Science Program; 40th Tuberculosis and Leprosy Research Conference. (Seattle, Washington, 2005).

43. Hess, J. et al. Mycobacterium bovis bacille CalmetteGuérin strains secreting listeriolysin of Listeria monocytogenes. Proc. Natl Acad. Sci. USA 95 5299-5304 (1998)

44. Albert, M. L., Sauter, B. \& Bhardwaj, N. Dendritic cells acquire antigen from apoptotic cells and induce class I-restricted CTLs. Nature 392, 86-89 (1998).

45. Nasser Eddine, A. \& Kaufmann, S. H. E. Improved protection by recombinant BCG. Microbes Infect. 7 939-946 (2005)

46. Grode, L. et al. Increased vaccine efficacy against tuberculosis of recombinant Mycobacterium bovis bacille Calmette-Guérin mutants that secrete listeriolysin. J. Clin. Invest. 115, 2472-2479 (2005).

47. Kaufmann, S. H. E. Rational vaccine design against tuberculosis. Presented at New Approaches to Vaccin Development. (Sept. 8-10, Berlin, Germany, 2005). 
48. Laufer, A. Managing the transition from academic vaccine research to license oriented development. Presented at New Approaches to Vaccine Development. (Sept. 8-10, Berlin, Germany, 2005).

49. Portnoy, D. A. et al. Capacity of listeriolysin O, streptolysin $\mathrm{O}$, and perfringolysin $\mathrm{O}$ to mediate growth of Bacillus subtilis within mammalian cells. Infect. Immun. 60, 2710-2717 (1992).

50. Edwards, K. M. et al. Iron-cofactored superoxide dismutase inhibits host responses to Mycobacterium tuberculosis. Am. J. Respir. Crit. Care Med. 164 2213-2219 (2001)

51. Kernodle, D. S. Pro-apoptotic BCG vaccine that enhances cellular immune responses and reduces tissue damage. Aeras Global TB Vaccine Foundation Scott Thaler Lecture Series. (Bethesda, Maryland, 2004).

52. Jacobs, B. Live attenuated vaccines for TB: Mining the immune evasion function of the tubercle bacillus. Presented at New Approaches to Vaccine Development. (Sept. 8-10,Berlin, Germany, 2005).

53. Chattergoon, M. A. et al. Targeted antigen delivery to antigen-presenting cells including dendritic cells by engineered Fas-mediated apoptosis. Nature Biotechnol. 18, 974-979 (2000).

54. Sheridan, J. P. et al. Control of TRAIL-induced apoptosis by a family of signaling and decoy receptors. Science 277, 818-821 (1997).

55. Brosch, R. et al. Comparative genomics of the mycobacteria. Int. J. Med. Microbiol. 290, 143-152 (2000).

56. Mahairas, G. G. et al. Molecular analysis of genetic differences between Mycobacterium bovis BCG and virulent $M$. bovis. J. Bacteriol. 178, 1274-1282 (1996).

57. Pym, A. S. et al. Recombinant BCG exporting ESAT-6 confers enhanced protection against tuberculosis. Nature Med. 9, 533-539 (2003).

58. Pym, A. S. et al. Loss of RD1 contributed to the attenuation of the live tuberculosis vaccines Mycobacterium bovis BCG and Mycobacterium microti. Mol. Microbiol. 46, 709-717 (2002).

59. Behr, M. A. et al. Comparative genomics of BCG vaccines by whole-genome DNA microarray. Science 284, 1520-1523 (1999)

60. Gordon, S. V. et al. Identification of variable regions in the genomes of tubercle bacilli using bacterial artificia chromosome arrays. Mol. Microbiol. 32, 643-645 (1999).

61. Salamon, H. et al. Detection of deleted genomic DNA using a semiautomated computational analysis of GeneChip data. Genome Res. 10, 2044-2054 (2000).

62. Kamath, A. T. et al. In New Live Mycobacterial Vaccines: Defining Essential Steps Towards Clinical Development. (WHO, Geneva, Switzerland, 2004).

63. Soto, C. Y. et al. IS6110 mediates increased transcription of the $p h o P$ virulence gene in a multidrug-resistant clinical isolate responsible for tuberculosis outbreaks. J. Clin. Microbiol. 42, 212-219 (2004)

64. Perez, E. et al. An essential role for phoP in Mycobacterium tuberculosis virulence. Mol. Microbiol. 41, 179-187 (2001).

65. Guleria, I. et al. Auxotrophic vaccines for tuberculosis. Nature Med. 2, 334-337 (1996)

66. Smith, D. A. et al. Characterization of auxotrophic mutants of Mycobacterium tuberculosis and their potential as vaccine candidates. Infect. Immun. 69 1142-1150 (2001)

67. Jackson, M. et al. Persistence and protective efficacy of a Mycobacterium tuberculosis auxotroph vaccine. Infect. Immun. 67, 2867-2873 (1999).

68. Sambandamurthy, V. K. et al. Long-term protection against tuberculosis following vaccination with a severely attenuated double lysine and pantothenate auxotroph of Mycobacterium tuberculosis. Infect. Immun. 73, 1196-1203 (2005)

69. Sambandamurthy, V. K. et al. A pantothenate auxotroph of Mycobacterium tuberculosis is highly attenuated and protects mice against tuberculosis. Nature Med. 8, 1171-1174 (2002)

70. Sambandamurthy, V. K. \& Jacobs, W. R. Live attenuated mutants of Mycobacterium tuberculosis as candidate vaccines against tuberculosis. Microbes Infect. 7, 955-961(2005).

71. Andersen, A. B. \& Hansen, E. B. Structure and mapping of antigenic domains of protein antigen $b, a$ 38,000-molecular-weight protein of Mycobacterium tuberculosis. Infect. Immun. 57, 2481-2488 (1989).
72. Nagai, S. et al. Isolation and partial characterization of major protein antigens in the culture fluid of Mycobacterium tuberculosis. Infect. Immun. $\mathbf{5 9}$ 372-382 (1991).

73. Young, D. B. \& Garbe, T. R. Lipoprotein antigens of Mycobacterium tuberculosis. Res. Microbiol. 142 55-65 (1991)

74. Andersen, P. et al. Recall of long-lived immunity to Mycobacterium tuberculosis infection in mice. J. Immunol. 154, 3359-3372 (1995).

75. Skjot, R. L. et al. Comparative evaluation of lowmolecular-mass proteins from Mycobacterium tuberculosis identifies members of the ESAT- 6 family as immunodominant T-cell antigens. Infect. Immun. 68, 214-220 (2000).

76. Alderson, M. R. et al. Expression cloning of an immunodominant family of Mycobacterium tuberculosis antigens using human $\mathrm{CD} 4^{+} \mathrm{T}$ cells J. Exp. Med. 191, 551-560 (2000).

77. Dillon, D. C et al. Molecular and immunological characterization of Mycobacterium tuberculosis CFP-10, an immunodiagnostic antigen missing in Mycobacterium bovis BCG. J. Clin. Microbiol. 38 , 3285-3290 (2000)

78. Skeiky, Y. A. et al. T-cell expression cloning of a Mycobacterium tuberculosis gene encoding a protective antigen associated with the early control of infection. J. Immunol. 165, 7140-7149 (2000).

79. Laal, S. \& Skeiky, Y. A. W. In Tuberculosis and the Tubercle Bacillus. (eds Cole, S. T. et al.) 71-83 (American Society for Microbiology Press, Washington DC, 2005).

80. Skeiky, Y. A. et al. Cloning, expression, and immunological evaluation of two putative secreted serine protease antigens of Mycobacterium tuberculosis. Infect. Immun. 67, 3998-4007 (1999).

81. Dillon, D. C. et al. Molecular characterization and human T-cell responses to a member of a novel Mycobacterium tuberculosis mtb39 gene family. Infect. Immun. 67, 2941-2950 (1999).

82. Covert, B. A. et al. The application of proteomics in defining the T-cell antigens of Mycobacterium tuberculosis. Proteomics 1, 574-586 (2001)

83. Mattow, J. et al. Comparative proteome analysis of culture supernatant proteins from virulent Mycobacterium tuberculosis H37Rv and attenuated M. bovis BCG Copenhagen. Electrophoresis 24 3405-3420 (2003)

84. Mollenkopf, H. J. et al. Application of mycobacterial proteomics to vaccine design: improved protection by Mycobacterium bovis BCG prime-Rv3407 DNA boost vaccination against tuberculosis. Infect. Immun. 72, 6471-6479 (2004)

85. Huygen, K. et al. Immunogenicity and protective efficacy of a tuberculosis DNA vaccine. Nature Med. 2 893-898 (1996)

86. Baldwin, S. L. et al. Evaluation of new vaccines in the mouse and guinea pig model of tuberculosis. Infect. Immun. 66, 2951-2959 (1998).

87. Skjot, R. L. et al. Epitope mapping of the immunodominant antigen TB10.4 and the two homologous proteins TB10.3 and TB12.9, which constitute a subfamily of the esat- 6 gene family. Infect. Immun. 70, 5446-5453 (2002).

88. Skeiky, Y. A. et al. Differential immune responses and protective efficacy induced by components of a tuberculosis polyprotein vaccine, Mtb72F, delivered as naked DNA or recombinant protein. J. Immunol. 172, 7618-7628 (2004).

89. Weinrich Olsen, A. et al. Protection of mice with a tuberculosis subunit vaccine based on a fusion protein of antigen $85 \mathrm{~b}$ and ESAT-6. Infect. Immun. 69 2773-2778 (2001)

90. Brandt, L. et al. The protective effect of the Mycobacterium bovis BCG vaccine is increased by coadministration with the Mycobacterium tuberculosis 72-kilodalton fusion polyprotein Mtb72F in $M$. tuberculosis-infected guinea pigs. Infect. Immun. 72, 6622-6632 (2004)

91. Olsen, A. W. et al. Efficient protection against Mycobacterium tuberculosis by vaccination with a single subdominant epitope from the ESAT- 6 antigen. Eur. J. Immunol. 30, 1724-1732 (2000).

92. Olsen, A. W. et al. Protective effect of a tuberculosis subunit vaccine based on a fusion of antigen $85 \mathrm{~B}$ and ESAT-6 in the aerosol guinea pig model. Infect. Immun. 72, 6148-6150 (2004).

93. Mazurek, G. H. et al. Comparison of a whole-blood IFN- $\gamma$ assay with tuberculin skin testing for detecting latent Mycobacterium tuberculosis infection. JAMA 286, 1740-1747 (2001).
94. Mazurek, G. H. \& Villarino, M. E. Guidelines for using the QuantiFERON-TB test for diagnosing latent Mycobacterium tuberculosis infection. Centers for Disease Control and Prevention. MMWR Recomm. Rep. 52, 15-18 (2003).

95. Dietrich, J. et al. Exchanging ESAT-6 with TB10. 4 in an Ag85B fusion molecule-based tuberculosis subunit vaccine: efficient protection and ESAT-6-based sensitive monitoring of vaccine efficacy. J. Immunol. 174, 6332-6339 (2005).

96. Brooks, J. V. et al. Boosting vaccine for tuberculosis. Infect. Immun. 69, 2714-2717 (2001).

97. Goonetilleke, N. P. et al. Enhanced immunogenicity and protective efficacy against Mycobacterium tuberculosis of bacille CalmetteGuérin vaccine using mucosal administration and boosting with a recombinant modified vaccinia virus Ankara. J. Immunol. 171, 1602-1609 (2003).

98. Wang, J. et al. Single mucosal, but not parenteral immunization with recombinant adenoviral-based vaccine provides potent protection from pulmonary tuberculosis. J. Immunol. 173, 6357-6365 (2004).

99. Vogels, R. et al. Replication-deficient human adenovirus type 35 vectors for gene transfer and vaccination: efficient human cell infection and bypass of pre-existing adenovirus immunity. J. Virol. 77, 8263-8271 (2003)

100. Havenga et al. Novel replication-incompetent adenoviral B-group vectors. J. Virol. (In the press)

101. Hone, D. Optimization of nucleic acid vaccine delivery by bacterial vectors. Presented at New Approaches to Vaccine Development. (Sept. 8-10, Berlin, Germany, 2005)

102. Schnappinger, D. et al. Transcriptional adaptation of Mycobacterium tuberculosis within macrophages: insights into the phagosomal environment. J. Exp. Med. 198, 693-704 (2003).

103. Voskuil, M. I. et al. Inhibition of respiration by nitric oxide induces a Mycobacterium tuberculosis dormancy program. J. Exp. Med. 198, 705-713 (2003).

104. Capuano, S. V. et al. Experimental Mycobacterium tuberculosis infection of cynomolgus macaques closely resembles the various manifestations of human M. tuberculosis infection. Infect. Immun. $\mathbf{7 1}$, 5831-5844 (2003).

105. Hussey, G., Hawkridge, T., Geiter, L. \& Hanekom, W. Presented at TB vaccines for the world (April 19-21, Vienna, Austria, 2006).

106. McShane, H. et al. Recombinant MVA85A boosts BCG-primed and naturally acquired antimycobacterial immunity in humans. Nature Med. 10, 1240-1244 (2004).

107. Radosevic, K. et al. Presented at TB vaccines for the world (April 19-21, Vienna, Austria, 2006).

\section{Acknowledgements}

We are grateful to Dr. L. Barker for his input and critical reading of this article. The Aeras Global TB Vaccine Foundation is supported by a major grant from the Bill and Melinda Gates Foundation.

Competing interests statement

The authors declare no competing financial interests.

\section{DATABASES}

The following terms in this article are linked online to: Entrez Gene: http://www.ncbi.nlm.nih.gov/entrez/query. fcgi?db=gene

lysA | panC | panD |Rv3238c|ureC

Entrez Genome Project: http://www.ncbi.nlm.nih.gov/

entrez/query.fcgi?db=genomeprj

Listeria monocytogenes |Mycobacterium bovis |

Mycobacterium tuberculosis

UniProtKB: http://ca.expasy.org/sprot

Ag85A|Ag85B | DnaK | GroES | Hly | IFN- $\gamma$ | MPT32 |MPT46 |

MPT53 | MPT63 | Mtb9.9 | Mtb32 | Phop | TB10.4

FURTHER INFORMATION

Author's homepage: www.ae

CDC HIV/AIDS Prevention:

http://www.cdc.gov/hiv/dhap.htm

South African TB Vaccine Initiative:

http://www.satvi.uct.ac.za

TubercuList: http://genolist.pasteur.fr./TubercuList

Access to this links box is available online. 